\title{
Predictors of Nerve Stimulator Success in Patients With Overactive Bladder
}

\author{
Kristian D. Stensland ${ }^{1}$, Bennett Sluis ${ }^{2}$, Jay Vance ${ }^{2}$, Jared P. Schober ${ }^{2}$, Lara S. MacLachlan ${ }^{1}$, Arthur P. Mourtzinos ${ }^{1}$ \\ ${ }^{1}$ Lahey Hospital and Medical Center, Burlington, MA, USA \\ ${ }^{2}$ Tufts University School of Medicine, Boston, MA, USA
}

Purpose: To identify factors associated with successful sacral nerve stimulator (SNS) trial after SNS implantation for the treatment of medication refractory overactive bladder $(\mathrm{OAB})$.

Methods: Patients undergoing treatment for OAB at Lahey Hospital and Medical Center between 2004 and 2016 were identified. Patients undergoing SNS placement were identified; SNS success was defined as permanent implantation of the SNS. Demographic, clinical and treatment data were extracted from patient charts; uni- and multivariate analyses were conducted to identify factors associated with SNS treatment success.

Results: A total of 128 patients were included. On univariate analysis, male sex, prior diagnosis of benign prostatic hyperplasia, and lower volume at first urge on urodynamics (UDS) were associated with unsuccessful SNS trial. On multivariate analysis, male sex (odds ratio [OR], 0.145; 95\% confidence interval [CI], 0.036-0.530) and lower volume at first urge on UDS (OR, $0.982 ; 95 \%$ CI, 0.967-0.995) were associated with unsuccessful SNS trial. A threshold value of $100 \mathrm{~mL}$ at first urge during preoperative UDS had a specificity of 0.86 in predicting SNS success in men.

Conclusions: SNS is frequently successful at relieving OAB symptoms. Male patients and those with lower volumes at first urge on UDS, particularly below $100 \mathrm{~mL}$, are more likely to have an unsuccessful SNS trial. Patients in these groups should be counseled on the lower likelihood of SNS success.

Keywords: Nerve stimulator; Botulinum toxins; Overactive urinary bladder; Urinary incontinence

- Research Ethics: This study was approved by the Institutional Review Board of Lahey Hospital and Medical Center. Written informed consent by the patients was waived by the IRB due to a retrospective nature of our study.

- Conflict of Interest: No potential conflict of interest relevant to this article was reported.

\section{INTRODUCTION}

Overactive bladder afflicts an estimated 9\%-16\% of patients per year in the United States alone [1]. With potentially devastating effects on quality of life, the burden of this disorder may have effects including more medical visits, more medication use, increased anxiety and depression, and an increase in both direct costs of medical care and indirect economic costs sec- ondary to missed work and decreased productivity.

At present, first line therapy for overactive bladder is behavioral intervention including life style modifications such as fluid management, bladder retraining, weight loss, smoking cessation, and pelvic floor muscle training. If these measures do not result in sufficient symptomatic improvement, patients are trialed on medical therapy including antimuscarinics or beta-3 adrenergic agonists. If these measures fail, patients can elect for

Corresponding author: Kristian D. Stensland (D) https://orcid.org/0000-0002-4917-2984 Department of Urology, Lahey Hospital and Medical Center, 41 Burlington Mall Road, Burlington, MA 01805, USA

E-mail: Kristian.stensland@lahey.org / Tel: +1-781-504-9387 / Fax: +1-781-744-5100 Submitted: April 15, 2018 / Accepted after revision: July 9, 2018 
other interventions such as botulinum toxin A injections, peripheral tibial nerve stimulation (PTNS), or sacral neuromodulation (sacral nerve stimulator, SNS) [2].

Sacral neuromodulation via an implanted stimulator has shown good response rates in patients with $\mathrm{OAB}$, with at least $50 \%$ improvement in symptoms in an estimated $67 \%-87 \%$ of patients [3]. The 2-stage nature of the procedure allows for a trial period of the nerve stimulator. The lead remains in place for up to 2 weeks to test for symptomatic improvement. If symptoms significantly improve during this initial trial, the permanent device is implanted. While the stimulator is highly effective in those for whom the modulator is permanently implanted, there are few methods currently available for predicting success of implantation prior to placement of the leads. Prior to the trial period, improved prediction of stimulator success would aid in patient counseling and treatment selection. Patients in whom a device is unlikely to be successful can be counseled to pursue other means of intervention without undergoing implantation of the trial lead.

In the present study, we utilize a database of tertiary treatments for overactive bladder to identify factors associated with successful stimulator trial resulting in permanent implantation of the modulator device.

\section{MATERIALS AND METHODS}

Patients undergoing treatment for $\mathrm{OAB}$ at Lahey Hospital and Medical Center between 2004 and 2016 were identified. For the overall database, any patient undergoing implantation of a SNS (first or second stage) or injection of botulinum toxin were included. For the present study, only information from patients undergoing implantation of a SNS was extracted. Demographic, clinical and treatment data were extracted from patient charts. Patients were included in the present study if they had complete data including preoperative urodynamics (UDS) data. Patients were included if they had SNS placement, data available on treatment success and clinical variables, and had undergone preoperative urodynamic studies.

Patients at our institution are offered a SNS after they have proven to have overactive bladder symptoms refractory to medical therapy. All patients who had a SNS placed between 2004 and 2016 were eligible for inclusion in the present study if they had complete pre- and postoperative clinical data.

All SNS procedures were performed by a single experienced surgeon. The SNS trials were performed in a staged manner, with the first stage performed in the operating room and tested with implantation of tined leads on a single side.

Success was defined as permanent implantation of the nerve stimulator at the scheduled surgical follow-up, with failure defined as removal of the SNS lead. For urinary tract infections within 180 days of surgery, the date of last positive culture was recorded and compared to the date of first stage implantation.

Univariate analyses were conducted to identify factors associated with SNS treatment success using chi-square, independent sample t-test, or Wilcox rank-sum statistics as appropriate. A multivariate logistic regression model using the significant and a priori clinical factors to predict SNS treatment success was also created. As a secondary subset analysis, patients were stratified by patient sex and analyses were rerun within each patient sex stratum. Significance was defined at the $\alpha=0.05$ level.

To identify an optimal predictive cutoff for volume at first urge and successful stimulator implantation, a receiver operating characteristic (ROC) curve was constructed utilizing the pROC package [4]. The 'best' balance between sensitivity and specificity was identified by Youden's index. Volumes were then dichotomized by a rounded volume for purposes of sensitivity and specificity calculation in a clinically-applicable cutoff. Statistical analyses were performed in $\mathrm{R}$ ver. 3.4.3 (R Foundation for Statistical Computing, Vienna, Austria) [5].

Study data were collected and managed using Research Electronic Data Capture (REDCap) electronic data capture tools hosted at Tufts University Medical Center and Lahey Hospital and Medical Center. REDCap is a secure, web-based application designed to support data capture for research studies, providing: (1) an intuitive interface for validated data entry; (2) audit trails for tracking data manipulation and export procedures; (3) automated export procedures for seamless data downloads to common statistical packages; and (4) procedures for importing data from external sources.

\section{RESULTS}

Of 276 total patients in the $\mathrm{OAB}$ database, 128 patients met inclusion criteria. Of these, 99 (77.3\%) had treatment success. Baseline clinical and urodynamic characteristics are detailed in Tables 1 and 2, respectively. Sex-specific clinical and urodynamic characteristics are available in supplementary tables. On univariate analysis, male sex, diagnosis of benign prostatic hyperplasia (BPH) and lower volume at first urge during UDS were associated with an unsuccessful SNS trial (Tables 1,2). On multivariate analysis, male sex (odds ratio [OR], 0.145; 95\% 
Table 1. Baseline characteristics in successful and unsuccessful implantation groups

\begin{tabular}{|c|c|c|c|}
\hline Characteristic & $\begin{array}{c}\% \\
\text { Successful }\end{array}$ & $\begin{array}{c}\% \\
\text { Unsuccessful }\end{array}$ & P-value \\
\hline Female sex & 73.7 & 27.6 & $<0.01$ \\
\hline Diabetes & 21.2 & 24.1 & 0.93 \\
\hline Hypertension & 54.5 & 44.8 & 0.48 \\
\hline Hyperlipidemia & 56.6 & 48.3 & 0.56 \\
\hline Multiple sclerosis & 2.0 & 0 & 1.00 \\
\hline Cerebral palsy & 1.0 & 0 & 1.00 \\
\hline Spinal cord injury & 6.1 & 0 & 0.39 \\
\hline Peripheral neuropathy & 5.1 & 3.4 & 1.00 \\
\hline Epilepsy & 4.0 & 6.9 & 0.89 \\
\hline History of UTI & 6.1 & 6.9 & 1.00 \\
\hline UTI within 180 days of surgery & 4.0 & 0 & 0.62 \\
\hline History of TURP & 3.0 & 6.9 & 0.69 \\
\hline Atrophic vaginitis & 10.1 & 3.4 & 0.45 \\
\hline History of hysterectomy & 20.2 & 10.3 & 0.35 \\
\hline Benign prostatic hyperplasia & 11.1 & 31.0 & 0.02 \\
\hline Erectile dysfunction & 10.1 & 13.8 & 0.82 \\
\hline Depression & 39.4 & 20.7 & 0.10 \\
\hline Anxiety & 15.2 & 13.8 & 1.00 \\
\hline Hypothyroidism & 10.1 & 13.8 & 0.82 \\
\hline Fibromyalgia & 3.0 & 0 & 0.80 \\
\hline Stroke/CVA & 6.1 & 10.3 & 0.70 \\
\hline Fecal incontinence & 8.1 & 0 & 0.25 \\
\hline Constipation & 13.1 & 6.9 & 0.55 \\
\hline Irritable bowel syndrome & 11.1 & 3.4 & 0.38 \\
\hline Interstitial cystitis & 2.0 & 3.4 & 1.00 \\
\hline Antimuscarinic use & 26.3 & 34.5 & 0.53 \\
\hline Beta agonist use & 8.1 & 6.9 & 1.00 \\
\hline Self-catheterization & 4.0 & 3.4 & 1.00 \\
\hline
\end{tabular}

UTI, urinary tract infection; TURP, transurethral resection of the prostate; CVA, cerebrovascular accident.

confidence interval $[\mathrm{CI}], 0.036-0.530 ; \mathrm{P} \leq 0.01)$ and volume at first urge on UDS (OR, 1.02; 95\% CI, 1.005-1.032; $\mathrm{P}=0.01$ ) were associated with unsuccessful SNS trial (Table 3).

A secondary analysis was performed stratified by patient sex. Within the male subgroup, 26 of 47 men (55.3\%) had successful treatment, compared to 73 of 81 women (90.1\%). The only factor significantly associated with SNS failure in the male group was mean volume at first urge on UDS $(80.5 \mathrm{~mL}$ in SNS
Table 2. Urodynamic characteristics of included subjects

\begin{tabular}{lccc}
\hline Urodynamic parameter & $\begin{array}{c}\text { Successful } \\
\text { (median) }\end{array}$ & $\begin{array}{c}\text { Unsuccessful } \\
\text { (median) }\end{array}$ & P-value \\
\hline Postvoid residual $(\mathrm{mL})$ & 6 & 0 & 0.17 \\
$\begin{array}{l}\text { Maximum cystometric } \\
\text { capacity }(\mathrm{mL})\end{array}$ & 250 & 225 & 0.86 \\
Maximum flow rate $(\mathrm{mL} / \mathrm{sec})$ & 11.7 & 8.7 & 0.13 \\
Volume at first urge $(\mathrm{mL})$ & 115.5 & 87 & $<0.01$ \\
$\begin{array}{l}\text { Maximum detrusor } \\
\text { pressure }\left(\mathrm{cm} \mathrm{H}_{2} \mathrm{O}\right)\end{array}$ & 24 & 30 & 0.09 \\
Volume voided $(\mathrm{mL})$ & 200 & 150 & 0.40 \\
\hline
\end{tabular}

Table 3. Multivariate logistic regression predicting successful SNS trial

\begin{tabular}{lccc}
\hline Factor & Odds ratio & $95 \%$ CI & P-value \\
\hline Age & 1.01 & $0.97-1.06$ & 0.51 \\
Volume at first urge (UDS) & 1.02 & $1.01-1.03$ & $<0.01$ \\
Male sex & 0.15 & $0.04-0.53$ & $<0.01$ \\
Benign prostatic hyperplasia & 1.52 & $0.35-6.80$ & 0.57 \\
\hline
\end{tabular}

SNS, sacral nerve stimulator; CI, confidence interval; UDS, urodynamics.

failure compared to $126.5 \mathrm{~mL}$ in $\mathrm{SNS}$ success, $\mathrm{P} \leq 0.01$ ). Similarly, on multivariate analysis only lower volume at first urge was statistically significantly associated with SNS failure (OR, 0.97; 95\% CI, 0.94-0.99) (Table 4).

Within the female group, there were no statistically significant associations between observed variables and SNS success. Notably, mean volume at first urge on UDS was not statistically significantly associated with SNS failure, though there was a similarly large clinical difference between groups $(97.5 \mathrm{~mL}$ in SNS failure compared to $136.0 \mathrm{~mL}$ in SNS success). On multivariate analysis in the female group, there were no significant factors associated with SNS success (Table 5).

A ROC curve for the sensitivity and specificity of volume at first urge for SNS failure produced an area under the curve of 0.70 . For both male and female sex considered together, volume at first urge of $101.5 \mathrm{~mL}$ was determined to be optimal as a threshold value. To facilitate clinical application, a volume of $100 \mathrm{~mL}$ was used as a threshold for dichotomization of high and low volume at first urge for all patients, which yielded a sensitivity of 0.83 and specificity of 0.49 for predicting nerve stimulator success for all patients. Applied only to male patients, the ROC had an area under the curve of 0.765 with an optimal threshold value of $107 \mathrm{~mL}$. Application of the $100-\mathrm{mL}$ 
Table 4. Characteristics in successful and unsuccessful implantation groups within male sex subset

\begin{tabular}{|c|c|c|c|}
\hline Factor & $\begin{array}{c}\% \\
\text { Successful }\end{array}$ & $\begin{array}{c}\% \\
\text { Unsuccessful }\end{array}$ & P-value \\
\hline Diabetes & 30.8 & 33.3 & 1.00 \\
\hline Hypertension & 73.1 & 57.1 & 0.40 \\
\hline Hyperlipidemia & 61.5 & 57.1 & 0.99 \\
\hline Spinal cord injury & 11.5 & 0 & 0.31 \\
\hline Peripheral neuropathy & 0 & 4.8 & 0.91 \\
\hline Epilepsy & 3.8 & 4.8 & 1.00 \\
\hline History of UTI & 3.8 & 4.8 & 1.00 \\
\hline History of TURP & 11.5 & 9.5 & 1.00 \\
\hline Benign prostatic hyperplasia & 42.3 & 42.9 & 1.00 \\
\hline Erectile dysfunction & 38.5 & 19.0 & 0.26 \\
\hline Depression & 34.6 & 14.3 & 0.21 \\
\hline Anxiety & 15.4 & 9.5 & 0.87 \\
\hline Hypothyroidism & 3.8 & 9.5 & 0.85 \\
\hline Stroke/CVA & 7.7 & 9.5 & 1.00 \\
\hline Fecal incontinence & 15.4 & 0 & 0.18 \\
\hline Constipation & 19.2 & 4.8 & 0.30 \\
\hline Irritable bowel syndrome & 7.7 & 4.8 & 1.00 \\
\hline Antimuscarinic use & 15.4 & 33.3 & 0.27 \\
\hline Beta agonist use & 15.4 & 9.5 & 0.87 \\
\hline Self-catheterization & 3.8 & 4.8 & 1.00 \\
\hline
\end{tabular}

UTI, urinary tract infection; TURP, transurethral resection of the prostate; CVA, cerebrovascular accident.

threshold yielded a sensitivity of 0.58 and specificity of 0.86 for SNS success in men.

\section{DISCUSSION}

In the management of overactive bladder, sacral nerve modulation via SNS holds a role as an effective tertiary intervention once implanted. Previously, attempts to identify predictors of SNS success have been primarily analyzed using long-term outcomes $[3,6,7]$. These outcomes are helpful for those patients who have successful implantation of the permanent lead and generator, and it is appropriate to quote the high success rates $(67 \%-87 \%)$ to patients once the stimulator is in place [3]. Patients unlikely to have successful trials, however, should be spared the initial implantation trial if possible. Avoiding an SNS trial prevents an unnecessary procedure, and saves providers, patients, and the healthcare system the accompanying costs.
Table 5. Characteristics in successful and unsuccessful implantation groups within female sex subset

\begin{tabular}{|c|c|c|c|}
\hline Factor & $\begin{array}{c}\% \\
\text { Successful }\end{array}$ & $\begin{array}{c}\% \\
\text { Unsuccessful }\end{array}$ & P-value \\
\hline Diabetes & 17.8 & 0 & 0.43 \\
\hline Hypertension & 47.9 & 12.5 & 0.12 \\
\hline Hyperlipidemia & 54.8 & 25.0 & 0.22 \\
\hline Multiple sclerosis & 2.7 & 0 & 1.00 \\
\hline Cerebral palsy & 1.4 & 0 & 1.00 \\
\hline Spinal cord injury & 4.1 & 0 & 1.00 \\
\hline Peripheral neuropathy & 6.8 & 0 & 1.00 \\
\hline Epilepsy & 4.1 & 12.5 & 0.86 \\
\hline History of UTI & 6.8 & 12.5 & 1.00 \\
\hline Atrophic vaginitis & 13.7 & 12.5 & 1.00 \\
\hline History of hysterectomy & 27.4 & 37.5 & 0.85 \\
\hline Depression & 41.1 & 37.5 & 1.00 \\
\hline Anxiety & 15.1 & 25.0 & 0.83 \\
\hline Hypothyroidism & 12.3 & 25.0 & 0.65 \\
\hline Fibromyalgia & 4.1 & 0 & 1.00 \\
\hline Stroke/CVA & 5.5 & 12.5 & 0.99 \\
\hline Fecal incontinence & 5.5 & 0 & 1.00 \\
\hline Constipation & 11.0 & 12.5 & 1.00 \\
\hline Irritable bowel syndrome & 12.3 & 0 & 0.64 \\
\hline Interstitial cystitis & 2.7 & 12.5 & 0.69 \\
\hline Antimuscarinic use & 30.1 & 37.5 & 0.98 \\
\hline Beta agonist use & 5.5 & 0 & 1.00 \\
\hline Self-catheterization & 4.1 & 0 & 1.00 \\
\hline
\end{tabular}

UTI, urinary tract infection; CVA, cerebrovascular accident.

There is a dearth of published research assessing potential predictors of SNS success, especially regarding procedural outcomes in male patients. In this study, we explore factors associated with successful implantation of SNS, including sex-specific stratified analysis, the first such analysis in the published literature to our knowledge.

Previously, there have only been limited investigations into predictors of success for nerve stimulator implantation. Van Balken et al. [8] explored prognostic factors for success of PTNS, and found mental health to be associated with successful reduction of symptoms via PTNS with worse mental health portending worse outcomes. The neural pathway for PTNS, while analogous to SNS, represents a distinct procedure. Yazdany et al. [9] did perform an analysis of female patients undergoing a 2-stage 
SNS procedure. In their analysis, 58 of 76 patients $(76 \%)$ had successful implantation of a permanent lead. Their only statistically significant predictor of trial success leading to permanent implantation was greater than 10 daily incontinence events compared to fewer than 5 daily incontinence events.

Overall, the SNS appears to be more effective in females than males, with nearly double the success rate in this series. With only one in ten females having explant of the SNS lead secondary to insufficient symptomatic improvement, it is reasonable to counsel female patients that they will most likely have a successful trial of the device. Moreover, there were no statistically significant associations between observed characteristics and SNS success in the female-only stratum. We were unable to find characteristics that might aid in identifying specifically female patients who are at higher risk of unsuccessful SNS trial. It is possible that there is no systematic factor among women that can predict success, or such a variable was unobserved in the present study.

Among men, the SNS trial was successful in roughly half of the cases observed. On both univariate and multivariate analysis within the male-only stratum, lower volume at first urge on preoperative UDS was the only statistically significant factor associated with unsuccessful SNS trial. Perhaps the most striking finding of our study is the high specificity (0.86) of a volume at first urge of $100 \mathrm{~mL}$ in male patients. This suggests that if a male patient has first urge at less than $100 \mathrm{~mL}$, he is highly unlikely to have a successful SNS trial. Given these findings, men should be counseled that SNS in men is much less successful than in women, and that there is a roughly a 50\% chance that their SNS lead will be explanted after the trial period. In particular, men with low volumes at first urge, particularly below $100 \mathrm{~mL}$, should be counseled that their SNS trial is unlikely to be successful.

In general, men with obstruction should have a bladder outlet procedure either in lieu of or in complement to overactive bladder treatment. Only men with mild, insignificant or no obstructive symptoms were offered nerve stimulator therapy. It is still interesting that men with documented $\mathrm{BPH}$ were found more frequently in the unsuccessful group; these men may benefit from other therapies prior to nerve stimulation trials.

There are limitations to the present study. Foremost, a relatively small sample size which should temper interpretation and generalizability of the results. Validation of the findings in this study will require larger studies incorporating more patients from multiple institutions and regions. Additionally, there were relatively few failures in female patients which may prevent proper elucidation of predictive factors for success in this sex subset. This study did not assess symptom improvement on a continuous (as opposed to dichotomous) or longer term scale; the outcome measure was only successful implantation of the permanent SNS system. While a definitive measure of symptomatic improvement would be useful in quantitatively assessing outcomes, the use of permanent stimulator implantation reflects a dichotomous success measure that is clinically applicable for practitioners in determining whether a procedure may be successful. In addition, the data in this study were also collected retrospectively from electronic medical records; patients were not directly interviewed and data were not collected prior to the initiation of analyses, which may bias results.

Future studies should more closely examine the role of urodynamics prior to SNS trial implantation, particularly in male patients, to corroborate these findings. Volume at first urge may be accompanied by additional clinical or cystometric factors in predicting SNS success, but further study is needed to confirm this finding and identify these other factors. Additionally, the pooling of multiple institutions' data may facilitate the more accurate prediction of failure in female patients and other subsets with both rarer utilization of SNS and low failure rates. Future studies would also benefit from accurately assessing the degree of symptomatic reduction and the duration of SNS success after 'permanent' implantation.

In conclusion, when counseling patients with overactive bladder for SNS implantation, men, and particularly men with volumes less than $100 \mathrm{~mL}$ at first urge on UDS, should be counseled that the likelihood of successful symptom relief and permanent stimulator implantation is significantly lower than the average female patient. Further study with more patients could identify factors associated with success in women, as well as confirm and expand our findings in men.

\section{AUTHOR CONTRIBUTION STATEMENT}

- Full access to all the data in the study and takes responsibility for the integrity of the data and the accuracy of the data analysis: $K D S, B S$, JV, JPS, LSM, APM

- Study concept and design: $A P M, L S M, K D S$

- Acquisition of data: $B S, J V, K D S$

- Analysis and interpretation of data: KDS

- Drafting of the manuscript: KDS, JV, BS

- Critical revision of the manuscript for important intellectual content: KDS, JPS, LSM, APM

- Statistical analysis: $K D S$ 
- Study supervision: $L S M, A P M$

\section{REFERENCES}

1. Eapen RS, Radomski SB. Review of the epidemiology of overactive bladder. Res Rep Urol 2016;8:71-6.

2. Gormley EA, Lightner DJ, Faraday M, Vasavada SP; American Urological Association; Society of Urodynamics, Female Pelvic Medicine. Diagnosis and treatment of overactive bladder (non-neurogenic) in adults: AUA/SUFU guideline amendment. J Urol 2015;193:1572-80.

3. Herbison GP, Arnold EP. Sacral neuromodulation with implanted devices for urinary storage and voiding dysfunction in adults. Cochrane Database Syst Rev 2009;(2):CD004202.

4. Robin X, Turck N, Hainard A, Tiberti N, Lisacek F, Sanchez JC, et al. pROC: an open-source package for $\mathrm{R}$ and $\mathrm{S}+$ to analyze and compare ROC curves. BMC Bioinformatics 2011;12:77.

5. The R Project for Statistical Computing [Internet]. Vienna (Aus- tria): The R Project for Statistical Computing; [cited 2018 Mar 15]. Available from: https://www.R-project.org/.

6. Olivera CK, Meriwether K, El-Nashar S, Grimes CL, Chen CC, Orejuela F, et al. Nonantimuscarinic treatment for overactive bladder: a systematic review. Am J Obstet Gynecol 2016;215:34-57.

7. Siddiqui NY, Amundsen CL, Visco AG, Myers ER, Wu JM. Costeffectiveness of sacral neuromodulation versus intravesical botulinum A toxin for treatment of refractory urge incontinence. J Urol 2009;182:2799-804.

8. van Balken MR, Vergunst H, Bemelmans BL. Prognostic factors for successful percutaneous tibial nerve stimulation. Eur Urol 2006; 49:360-5.

9. Yazdany T, Bhatia N, Nguyen J. Determining outcomes, adverse events, and predictors of success after sacral neuromodulation for lower urinary disorders in women. Int Urogynecol J 2011;22:154954. 